\title{
Pretransplant varicella vaccination is cost- effective in pediatric renal transplantation
}

Olson AD, Shope TC, Flynn JT. Pretransplant varicella vaccination is cost-effective in pediatric renal transplantation.

Pediatr Transplantation 2001: 5: 44-50. (C) Munksgaard, 2001

Abstract: Because of the severe complications that may result from varicella zoster virus (VZV) infection following renal transplantation (Tx), transplanted varicella-susceptible children exposed to varicella are typically given varicella zoster immunoglobulin (VZIG) as prophylaxis or are admitted and treated with parenteral acyclovir if VZIG prophylaxis fails. As both VZIG and hospitalization are costly, prevention of varicella infection by vaccination could potentially result in significant cost savings in addition to decreasing morbidity and mortality. To test this hypothesis, we developed a decision-analysis model to evaluate the cost-effectiveness of vaccinating patients with chronic renal failure (CRF) against varicella prior to renal transplant. Under baseline assumptions, vaccination for varicella pretransplant was a cost-effective strategy, with a cost of $\$ 211$ per patient vaccinated compared with $\$ 1,828$ per patient not vaccinated. The magnitude of cost savings from vaccination was sensitive to variations in the cost of varicella vaccine, the percentage of patients hospitalized for treatment with acyclovir, and the percentage of patients exposed to varicella infection. One- and two-way sensitivity analyses confirmed that vaccination was the dominant cost-effective strategy under all conditions examined. We conclude that vaccination for varicella pretransplant is cost-effective for patients with CRF, and that the magnitude of cost savings is sensitive to the cost of hospitalization, the percentage of patients exposed to varicella, and the cost of varicella vaccination. Pending results of ongoing studies of the safety and efficacy of VZV vaccine in children with CRF, we recommend that VZV vaccine be given to all children with $\mathrm{CRF}$.

\section{Allan D. Olson ${ }^{1}$, Thomas C. Shope ${ }^{2}$ and Joseph T. Flynn ${ }^{3}$}

Divisions of Pediatric ${ }^{1}$ Gastroenterology, ${ }^{2}$ Infectious Diseases and ${ }^{3}$ Nephrology, Department of

Pediatrics and Communicable Diseases, C.S. Mott Children's Hospital, University of Michigan, Ann Arbor, Michigan, USA

Key words: children - renal transplantation varicella - immunization - costs and cost analysis

Allan D. Olson, M.D., Centocor Inc., 200 Great Valley Parkway, Malvern, PA, 19355-1307, USA

Tel.: (610)-889-4741

Fax: (610)-651-6140

E-mail: olsona@centocor.com

Accepted for publication 30 June 2000
Varicella infection post-renal Tx may cause severe complications, including allograft rejection, hepatitis, pneumonitis, or death (1-12). Transplant recipients exposed to varicella are generally treated with VZIG in an attempt to prevent actual infection (13-16). Hospitalization for treatment with i.v. acyclovir is generally required in patients who develop varicella infection, despite

\footnotetext{
Abbreviations: CMV, cytomegalovirus; $\mathrm{CRF}$, chronic renal failure; EBV, Epstein-Barr virus; i.v., intravenous; NACHRI, National Association of Childrens' Hospitals and Related Institutions; Tx, transplantation; VZV, varicella zoster virus; VZIG, varicella zoster immunoglobulin.
}

VZIG prophylaxis, in order to decrease viral replication and prevent severe complications $(4,6$, $10,13,17-19)$. Varicella vaccination offers the possibility of decreasing the number of patients with varicella infection, and/or ameliorating the course of varicella infection, thereby preventing or reducing the costs associated with the usual approaches mentioned above $(9,10,14,20-27)$.

We hypothesized that prior to Tx, vaccination of all children with CRF who are susceptible to varicella infection is a cost-effective approach, resulting in decreased costs and improved outcomes, including decreased rates of hospital admissions, decreased requirements for VZIG prophylaxis, and a lower probability of death 
from varicella infection. In order to examine this hypothesis, we carried out a cost-analysis study, comparing the cost of universal screening by history and vaccination of VZV-negative patients with the standard treatments of VZIG administration post-exposure and hospitalization for treatment with i.v. acyclovir.

\section{Methods}

\section{Decision tree}

For this study we developed a decision-analysis tree using a decision-modeling program (Data 3.0; TreeAge, Inc., Cambridge, MA, USA). Our model compared two strategies: strategy 1 involved immunization of children with CRF against varicella prior to renal Tx; and strategy 2 involved no immunization. In strategy 2 (no immunization), non-VZV immune transplant recipients exposed to varicella were given VZIG for prophylaxis, and were hospitalized for treatment with parenteral acyclovir if active varicella infection developed despite prophylaxis.

Baseline assumptions and ranges for sensitivity analysis were obtained from a review of the literature, expert opinions, an estimate of costs of care at comparable Children's Hospitals, and clinical experience at the University of Michigan, and are outlined in Tables 1 and 2. Costs of the two-dose varicella vaccination program, VZIG administration, and hospitalization for i.v. acyclovir used in this model, were based upon costs reported in a national database generated by the NACHRI. Only patients without a prior history of varicella infection or vaccination were included in the model. A two-dose varicella vaccination program was chosen to ensure an optimal immune response in this immune-compromised population. The costs indicated in our baseline assumptions included all direct medical costs. For example, cost of immunization included the cost of two doses of vaccine, the cost of a visit, and the cost of vaccine administration.

The decision tree evaluated sequentially the impact of vaccination or lack of vaccination on:

1 the cost of treatment;

2 the use of VZIG;

3 the frequency of infection with varicella virus;

4 the number of hospital admissions; and

5 the number of deaths from varicella complications.

The frequency of infection was determined by the exposure rate to varicella, the risk of acquiring infection once exposed, the impact of receiving VZIG on the risk of infection, the risk of hospitalization as a result of infection, and the risk of death and allograft rejection from complications of varicella infection. In our model, we presumed that the risk of acquiring varicella infection once exposed was 0.5 , mid-range between the reported risk of acquiring infection for household exposures, which was as high as 0.85 , and the risk following a classroom exposure of 0.2 (28). The risk of exposure of 0.223 per year therefore resulted in a risk of infection of 0.111 per year. The risk of exposure of 0.223 was chosen so that the risk of infection rate approximated the average seroconversion rate of $11 \%$ per year reported in children aged $2-7 \mathrm{yr}(28,29)$. With a risk of infection with varicella of $11 \%$ per year up to $7 \mathrm{yr}$ of age, our model predicts that $45 \%$ of children would acquire varicella over the $5 \mathrm{yr}$ modeled period, compared with the reported acqusition rate of $55 \%$ for children aged $2-7 \mathrm{yr}$ (28-31).

For this analysis, all costs were discounted at $5 \%$ per year. Therefore, costs in the first year were assigned full value, while costs in subsequent years were discounted at an annual rate of $5 \%$ per year. It is a necessary and standard procedure to discount costs and savings that will occur in the future to adjust for the greater benefit of having a

Table 1. Probability assumptions used in the decision tree

\begin{tabular}{|c|c|c|c|c|}
\hline Event & Baseline & Worst case & Range & References/source of data \\
\hline Exposure to varicella per year & 0.223 & 0.16 & $0.16-0.248$ & (28) \\
\hline Varicella infection per year & 0.111 & 0.08 & $0.08-0.124$ & (28) \\
\hline Protection by varicella & 0.950 & 0.83 & $0.83-0.950$ & (29) \\
\hline Recognizing exposure to varicella & 0.800 & 0.95 & $0.70-0.950$ & ${ }^{*} \dagger$ \\
\hline Receiving VZIG once exposed & 0.900 & 0.95 & $0.50-0.950$ & ${ }^{*} \dagger$ \\
\hline Recognized protection by VZIG & 0.900 & 0.95 & $0.50-0.950$ & $\dagger(39)$ \\
\hline Sub-clinical infection & 0.060 & 0.10 & $0.04-0.150$ & $(31,30)$ \\
\hline
\end{tabular}

Probabilities are indicted as a decimal and represent probability per year.

* Expert opinion of transplant coordinators from Bone Marrow, Renal and Liver transplant services at the University of Michigan Medical Center.

$\uparrow$ Expert opinion of a Pediatric Infectious Disease Physician Panel at the University of Michigan Medical Center.

VZIG, varicella zoster immune globulin. 


\section{Olson et al.}

Table 2. Cost assumptions

\begin{tabular}{lllll}
\hline Costs & Baseline & Worst case & Range & Source of data \\
\hline Cost of immunization & $\$ 120$ & $\$ 200$ & $\$ 90-200$ & ${ }^{*} \dagger$ \\
Cost of VZIG treatment & $\$ 450$ & $\$ 400$ & $\$ 400-600$ & ${ }^{*} \dagger$ \\
Cost of hospital admission for acyclovir treatment & $\$ 7,200$ & $\$ 5,200$ & $\$ 5,200-7,200$ & ${ }^{*} \dagger$
\end{tabular}

Costs are expressed in US dollars and represent the cost per episode of treatment.

* Cost estimates from the University of Michigan.

$\uparrow$ Cost estimates from NACHRI Pediatric Hospital Cost Data.

VZIG, varicella zoster immune globulin.

resource (i.e. dollars to spend) today rather than at a future time.

To test the sensitivity of our analysis to changes in our assumptions, we examined the effect of varying our assumptions over a range of values indicated in Tables 2 and 3. In a 'one-way sensitivity analysis' each assumption was varied over the range indicated graphed on the $x$-axis and the effect on the cost of care under each analysis was noted on the $y$-axis. In a 'two-way sensitivity analysis' two assumptions were varied over the ranges simultaneously, and the most cost-effective strategy for each pair of inputs was determined.

\section{Results}

Cost savings from vaccination

Under our baseline assumptions, vaccination against varicella was the dominant cost-effective strategy, with a cost of $\$ 211$ per child vaccinated compared with $\$ 1,828$ per child not vaccinated (Fig. 1). Therefore for each dollar spent on the vaccination strategy, more than $\$ 8$ would be saved on avoided treatment costs.

In the immunization strategy, the major cost arose from primary prevention (varicella vaccination), while in the no-immunization strategy the greatest costs arose from secondary prevention (VZIG following exposure) and treatment (hospitalization for acyclovir following onset of varicella) (Fig. 2). The lower risk of varicella infection in the immunization strategy led to substantial savings from decreased hospital admissions and fewer patients requiring VZIG. These savings were considerably greater than the added cost of immunization (Fig. 2).
The likelihood of receiving VZIG was significantly greater under the no-immunization strategy $(64.8 \%)$ than under the immunization strategy $(0 \%)$ (Table 3$)$. The number of patients requiring hospitalization for treatment with i.v. acyclovir because of active varicella infection under the immunization strategy $(2.2 \%)$ was considerably less than under the no-immunization strategy $(42.3 \%)$.

\section{Sensitivity analysis}

The magnitude of cost savings from vaccination was sensitive to several variables, including:

- the cost of varicella vaccine;

- the percentage of patients exposed to varicella infection;

- the number of patients who successfully received and responded to VZIG with protection against varicella infection; and

- the percentage of patients hospitalized for treatment with acyclovir.

The cost saving (the difference in cost per case under the no-immunization strategy vs. the immunization strategy) rose as the cost of hospitalization increased (Fig. 3), as the rate of exposure to varicella increased (Fig. 4), and as the rate of protection from varicella vaccination increased (information not shown). The cost savings fell as the cost of vaccination increased (Fig. 5), the number of patients receiving VZIG increased (information not shown) and the efficacy of VZIG increased (information not shown). Although the magnitude of savings varied, the immunization strategy remained as a cost saving for all values considered. In addition, sensitivity analyses, changing both individual variables and multiple

Table 3. Outcomes under baseline assumptions

\begin{tabular}{lll}
\hline Outcomes & Immunized & Non-immunized \\
\hline Cost per patient & $\$ 250$ & \\
Probability of receiving VZIG & $0.0 \%$ & \\
Probability of infection & $2.8 \%$ & \\
Probability of hospitalization for acyclovir treatment & $2.2 \%$ & $45.0 \%$ \\
& $42.3 \%$ \\
\hline
\end{tabular}

Cost per patient and probabilities are indicated as the cost and chances for each patient. 


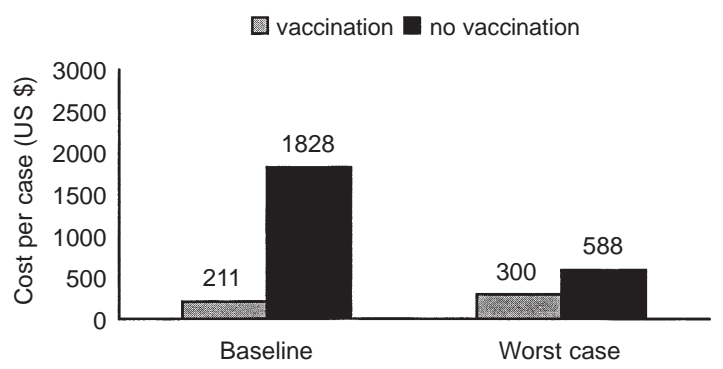

Fig. 1. Cost-effectiveness of vaccination. Cost per case under our baseline assumptions was significantly less $(\$ 211)$ than under the no-vaccination strategy $(\$ 1,828)$. Under our worst case, assumptions were chosen to limit the cost-effectiveness of the vaccination strategy $(\$ 300)$, as compared to the novaccination strategy $(\$ 588)$.

variables throughout the ranges indicated in Tables 2 and 3 , confirmed that vaccination remained the dominant cost-effective strategy under all conditions examined.

To illustrate the robust nature of our conclusion that vaccination for varicella is a cost saving, we developed a 'worst case' scenario where all the assumptions were chosen to prejudice the analysis against the varicella vaccination strategy. These assumptions were:

- efficacy of varicella vaccine of only $83 \%$;

- probability of exposure to varicella of $16 \%$ per year;

- recognition of varicella of $95 \%$;

- receipt of VZIG of $95 \%$;

- 95\% effectiveness of VZIG;

- cost of vaccination of $\$ 200$; and

- cost of hospitalization of $\$ 5,200$.

Even if all of these extreme conditions occured (all of which are prejudicial to the cost-effectiveness of the immunization strategy), varicella vaccination remained the most cost-effective strategy, with a cost of $\$ 300$ compared to a cost of no vaccinations of $\$ 588$ (Fig. 1). Therefore, even under these highly unlikely conditions, for

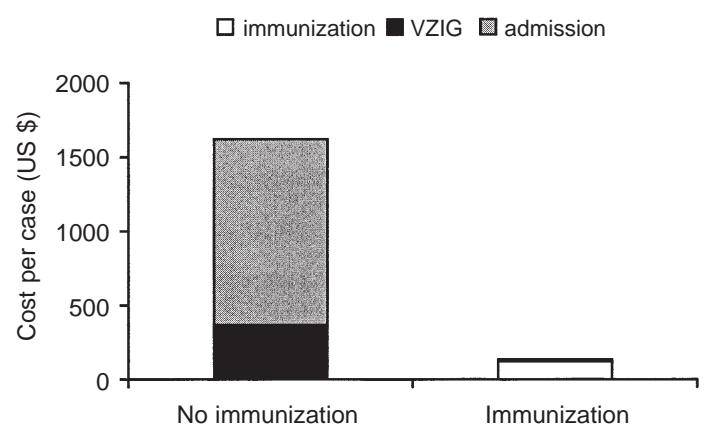

Fig. 2. Distribution of costs under the baseline assumptions. Under our baseline assumptions, the no-immunization strategy costs arose from hospitalization for treatment with acyclovir and varicella zoster immune globulin (VZIG) following exposure to varicella, and the immunization strategy cost arose predominantly from the cost of immunization.

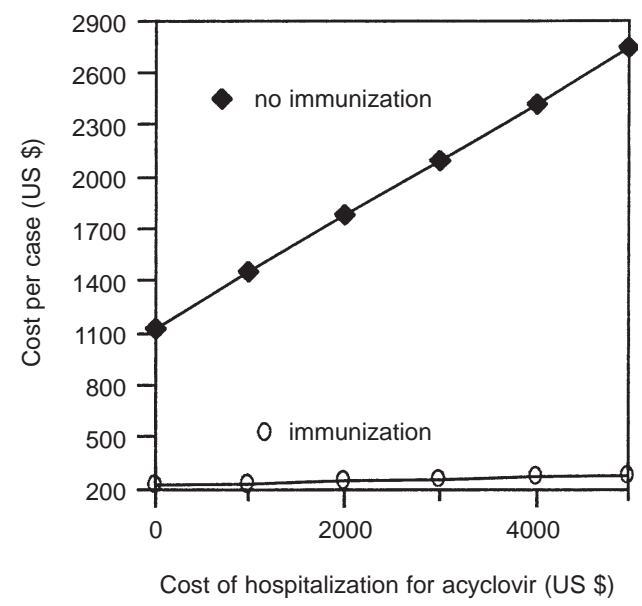

Fig. 3. Sensitivity analysis on the cost of hospitalization for treatment with acyclovir. As the cost for hospitalization for acyclovir increased, the cost of the no-immunization strategy rose, increasing the potential cost saving using the immunization strategy.

each dollar spent on varicella vaccination, almost $\$ 2$ would be saved on subsequent treatment costs.

\section{Discussion}

Following solid-organ Tx, viral infections remain an important source of morbidity in both children and adults, with the risk of most herpesvirus infections, in particular, beginning $\approx 4-6$ weeks post-Tx and continuing for the life of the transplant recipient (32). The risk of acquiring such infections is affected by the recipient's prior exposure to the virus and their overall state of immunosuppression, with recipients who have required increased amounts of immunosuppression because of rejection probably having an increased susceptibility for more severe disease

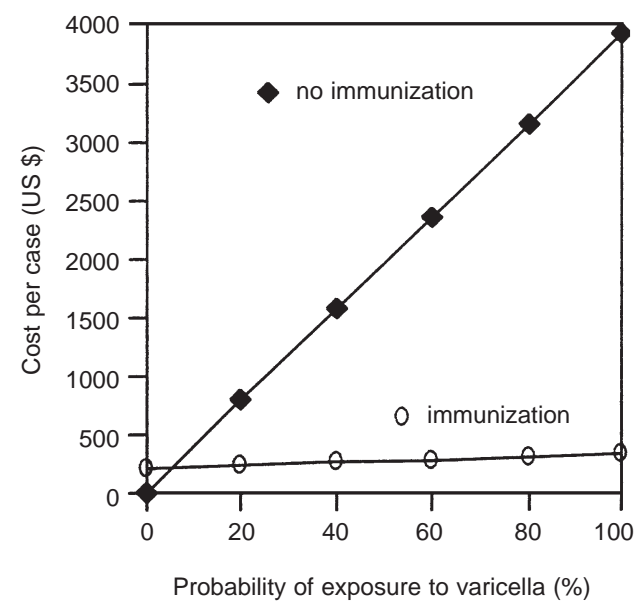

Fig. 4. Sensitivity analysis on probability of exposure to varicella. As the probability of exposure increased, the cost per case for the no-immunization strategy increased, raising the cost savings of the immunization strategy. 


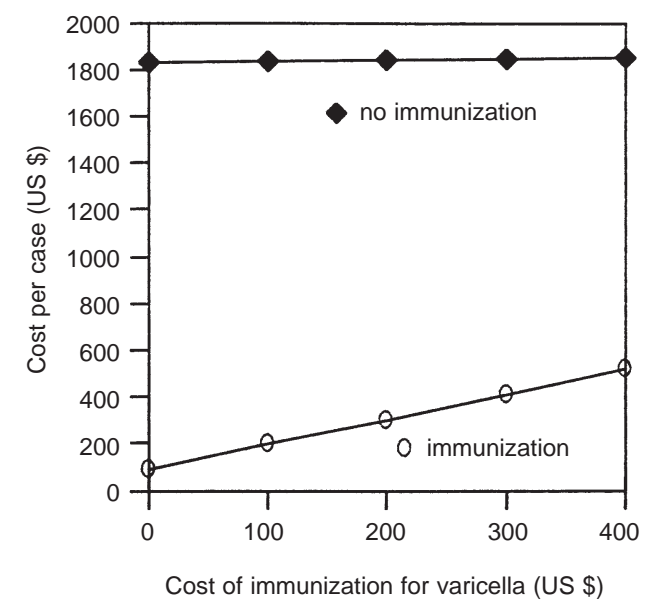

Fig. 5. Sensitivity analysis on the cost of immunization for varicella. As the cost of varicella vaccine rose, the cost per case under the immunization strategy rose slightly, decreasing slightly the cost savings of the immunization strategy.

compared to recipients without a history of rejection (33). For children, herpesvirus infections such as CMV, EBV, and VZV are of particular concern as many children will undergo Tx before they have been exposed to these agents $(19,34$, 35).

The complications of varicella infection postrenal Tx are well-documented, and include disseminated infection, acute renal failure, rejection, graft loss, and death $(1-13,19)$. Such complications may develop even with appropriate management using post-exposure VZIG prophylaxis, treatment with i.v. or oral acyclovir, and modification of immunosuppression $(6,9,10,19)$. Healthcare costs associated with post-transplant varicella infection can be substantial whether or not severe complications occur - the cost of administration of VZIG, which usually prevents the development of varicella infection $(15,16)$, can be as high as $\$ 450$ per treatment course. The costs of hospitalization are also high, at up to $\$ 7,200$ per admission.

Vaccination against varicella offers a potential strategy of not only preventing the morbidity associated with varicella infection, but also reducing healthcare costs (36). Varicella vaccine was licensed for use in the United States in March 1995, and since that time has been incorporated into the routine schedule of immunizations recommended by the Advisory Committee on Immunization Practices (27), American Academy of Pediatrics, Committee on Infectious Diseases (37), and other consensus bodies. Although the vaccine is generally well tolerated and quite effective, it has not been universally adopted by pediatricians and other primary care providers (38), which means that many children with CRF may not be immune to varicella when they are referred to Pediatric Nephrologists for dialysis or Tx. As the vaccine has been demonstrated to be safe and effective in children with CRF $(9,21,25)$, the opportunity exists to ensure that such children are varicella immune by the time they come to transplant $(14,20,26)$. Given that prior studies in children with renal transplants (22), as well as the early results of the Southwest Pediatric Nephrology Study Group Varivax ${ }^{\mathbb{R}}$ study (A. Furth, personal communication), have indicated that immunity to varicella will persist post-Tx, ensuring pretransplant immunity would appear to be an appropriate strategy for protection against posttransplant VZV infection.

In this study, we conducted a cost analysis of universal varicella vaccination of children with CRF prior to renal $\mathrm{Tx}$ in order to determine the cost efficacy of this practice. Although multiple assumptions were required in developing this model, our analysis demonstrated that varicella vaccination was the dominant strategy under all conditions anticipated, even under a worst-case scenario of decreased vaccine efficacy and low varicella exposure rates. Although the magnitude of cost savings was sensitive to the cost of hospitalization, the number of patients exposed to varicella virus, and the cost of varicella virus vaccination, vaccination was always the dominant cost-effective strategy, with savings ranging from $\$ 2$ to greater than $\$ 8$ per dollar spent on immunizations.

Given that the rate of varicella infection in non-immunized individuals is as high as 20 times that in immunized individuals $(40,43)$, and that varicella introduces the risk of graft rejection, a higher rate of graft rejection could be expected in non-immunized individuals. Widespread vaccination against VZV will potentially reduce the rate of infection and thus the rate of rejection, resulting in further cost savings as a result of decreased hospitalization and decreased use of expensive antirejection medications. If we had included these potential savings in our model, the immunization strategy would have shown even greater savings when compared with the no-immunization strategy.

We conclude that vaccination against varicella during the pretransplant period is costeffective for children with CRF. Our finding of the cost savings combined with the decreased risks of severe infection, graft rejection and other complications of post-transplant varicella add further weight to the call for complete vaccination of this population against varicella 
(26). Based on this analysis, we recommend that varicella vaccine be given to all children with chronic renal failure prior to $\mathrm{Tx}$.

\section{References}

1. Hurley JK, Greenslade T, Lewy PR, Ahmadian Y, FIRLIT C. Varicella-zoster infections in pediatric renal transplant recipients. Arch Surg 1980: 115: 751-752.

2. Peterson LR, Ferguson RM. Fatal central nervous system infection with varicella-zoster virus in renal trans-plant recipients. Transplantation 1984: 37 : 366-368.

3. Bourbigot B, Quillien MC, Airiau J, et al. Renal failure in the course of reactivation of varicella-zoster virus infection in a renal transplant recipient. J Infect 1986: 13: 159-162.

4. Bradley JR, Wreghitt TG, Evans DB. Chickenpox in adult renal transplant recipients. Nephrol Dial Transplant 1987: 1: 242-245.

5. Leguen E, Deminiere C, Larue JR, et al. Fatal varicella or Reye's syndrome: an unusual cause of death in a renal graft recipient. Nephrol Dial Transplant 1992: 7: 158-160.

6. Lynfield R, Herrin JT, Rubin RH. Varicella in pediatric renal transplant recipients. Pediatrics 1992: 90: 216-220.

7. Vadoud-Seyedi R, Liesnard C, Willaert F, Parent D. Fatal varicella in an immunocompromised adult. Dermatology 1993: 187: 47-49.

8. Bensousan TA, Moal MC, Vincent F, Nousbaum JB, Bour BIGOT B. Fulminant hepatitis revealing primary variella in a renal graft recipient. Transplant Proc 1995: 27: 2512.

9. Broyer M, Tete MJ, Guest G, Gagnadoux MF, Rouzioux C. Varicella zoster in children after kidney transplantation: long-term results of vaccination. Pediatrics 1997: 99: 35-39.

10. Kashtan CE, Cook M, Chavers BM, Mauer SM, Nevins TE. Outcome of chickenpox in 66 pediatric renal trans-plant recipients. J Pediatr 1997: 131: 874-877.

11. Rothwell WS, Gloor JM, Morgenstern BZ, Milliner DS. Disseminated varicella infection in pediatric renal transplant recipients treated with mycophenolate mofetil. Transplantation 1999: 68: 158-161.

12. Franco A, Marco P, Gil C, Muñoz C, Jiménez L. Fatal varicella with primary fibrinolysis in a renal transplant recipient. Nephrol Dial Transplant 1999: 14: 1291-1294.

13. Parnham AP, Flexman JP, Saker BM, Thatcher GN. Primary varicella in adult renal transplant recipients: a report of three cases plus a review of the literature. Clin Transpl 1995: 9: 115-118.

14. Tolkoff-Rubin NE, Rubin H. New strategies for the control of viral infection in organ transplantation. Clin Transpl 1995: 9: 255-259.

15. American Academy of Pediatrics. Varicella-Zoster Infections. In: Peter G., eds. 1997 Red Book: Report of the Committee on Infectious Diseases, 24th edn. Elk Grove Village, IL: American Academy of Pediatrics, 1997: 573-585.

16. Zayas E, Caraballo ZG, Otero LM, Delpín EAS. Varicella Zoster in a transplant program: experience with 15 cases and 70 contacts. Transplant Proc 1996: 28: 3296-3297.
17. Balfour HH Jr, McMonigal KA, Bean B. Acyclovir therapy of varicella-zoster virus infections in immunocompromised patients. J Antimicrob Chemother 1983: 12 (Suppl. B): 169-179.

18. Hayes K, Shakuntala V, Pingle A, Dhawan IK, Masri MA. Safe use of acyclovir (Zovirax) in renal transplant patients on cyclosporine A therapy: Case reports. Transplant Proc 1992: 24: 1926.

19. Furth SL, Sullivan EK, Neu AM, Tejani A, Fivush BA. Varicella in the first year after renal transplantation: a report of the North American Pediatric Renal Transplant Cooperative Study (NAPRTCS). Pediatr Transpl 1997: 1: $37-42$.

20. Gershon AA. Immunizations for pediatric transplant patients. Kidney Int 1993: 43: S87-S90.

21. Broyer M, Boudaillez B. Varicella vaccine in children with chronic renal insufficiency. Postgrad Med J 1985: 61 (Suppl. 4): 103-106.

22. Zamora I, Simon JM, Da Silva ME, Piqueras AI. Attenuated varicella virus vaccine in children with renal transplants. Pediatr Nephrol 1994: 8: 190-192.

23. Giacchino R, Marcellini M, Timitilli A, et al. Varicella vaccine in children requiring renal or hepatic transplantation. Transplantation 1995: 60: 1055-1056.

24. Furth Sl, Neu AM, Sullivan EK, Gensler G, Tejani A, FIVUSH BA. Immunization practices in children with renal disease: a report of the North American Pediatric Renal Transplant Cooperative Study. Pediatr Nephrol 1997: 11: 443-446.

25. Furth SL, Hogg R, Tarver J, Fivush BA. One year follow-up of Varivax ${ }^{\circledR}$ in children and adolescents with chronic renal insufficiency (CRI): a report of the Southwest Pediatric Nephrology Study Group. J Am Soc Nephrol 1999: 10: 164A.

26. Fivush BA, Neu AM. Immunization guidelines for pediatric renal disease. Semin Nephrol 1998: 18: 256-263.

27. Centers for Disease Control and Prevention. Prevention of Varicella: Updated recommendations of the Ad-visory Committee on Immunization Practices (ACIP). Morbid Mortal Wkly Rep 1999: 48: 1-5.

28. Finger R, Hughe JP, Meade BJ, Pelletier AR, Palmer CT. Age-specific incidence of chickenpox. Public Health Rep 1994: 109: 750-755.

29. Halloren Me, Cochi Sl, Lieu TA, Wharton M, Fenrs L. Theoretical epidemiologic and morbidity effects of routine varicella immunization of preschool children in the United States. Am J Epidemiol 1994: 140: 81-104.

30. Steele RW, Coleman MA, Fiser M. Varicella zoster in hospital personnel: skin test reactivity to monitor susceptibility. Pediatrics 1982: 70: 604-608.

31. Weibel RE, Neff BJ, Kuter BJ, et al. Live attenuated varicella virus vaccine: efficacy trial in healthy children. $\mathrm{N}$ Engl J Med 1984: 310: 1409-1415.

32. Fishman JA, Rubin RH. Infection in organ-transplant recipients. N Engl J Med 1998: 338: 1741-1751.

33. Klauser R, Zlabinger GJ, Traindl Franz M, et al. Influence of immunosuppressive therapy on infectious complications in renal transplant recipients. Transplant Proc 1992: 24: 292-294.

34. Ho M. Epidemiology of cytomegalovirus infection in man. In: Ho M, ed. Cytomegalovirus: Biology and Infection, 2nd edn. New York: Plenum Publishing, 1991: 155-187.

35. Evans AS, Niederman JC. Epstein-Barr virus. In: Evans AS, ed. Viral Infections of Humans: Epidem- 


\section{Olson et al.}

iology and Control. New York: Plenum Publishing, 1989: 265-292.

36. Kitai IC, King S, Gafni A. An economic evaluation of varicella vaccine for pediatric liver and kidney transplant recipients. Clin Infect Dis 1993: 17: 441-447.

37. American Academy of Pediatrics Committee on Infectious Diseases. Recommendations for the use of live attunated varicella vaccine.Pediatrics 1995: 95: 791-796.

38. Committee on Infectious Diseases. Varicella vaccine update. Pediatrics 2000: 105: 136-141.

39. WiNSNES R. Efficacy of zoster immunoglobulin in prophylaxis of varicella in high-risk patients. Acta Paediatr Scand 1978: 67: 72-82. 